\title{
TRANSCENDENCE OF GENERATING FUNCTIONS OF WALKS ON THE SLIT PLANE
}

\author{
MARTIN RUBEY
}

\begin{abstract}
Consider a single walker on the slit plane, that is, the square grid $\mathbb{Z}^{2}$ without its negative $x$-axis, who starts at the origin and takes his steps from a given set $\mathfrak{S}$. Mireille Bousquet-Mélou conjectured that - excluding pathological cases - the generating function counting the number of possible walks is algebraic if and only if the walker cannot cross the negative $x$-axis without touching it. In this paper we prove a special case of her conjecture.
\end{abstract}

\section{INTRODUCTION}

Let $\mathfrak{S}$ - the set of steps - be a finite subset of $\mathbb{Z}^{2}$. A walk on the slit plane is a sequence $(0,0)=w_{0}, w_{1}, \ldots, w_{n}$ of points in $\mathbb{Z}^{2}$, such that the difference of two consecutive points $w_{i+1}-w_{i}$ belongs to the set of steps $\mathfrak{S}$ and none of the points but the first lie on the half-line $\{(x, 0): x \leq 0\}$. An example for such a walk with set of steps

$$
\mathfrak{S}=\{(-1,-2),(-1,1),(-1,2),(1,-2),(1,1),(1,2)\}
$$

is shown in Figure 10

Recall that a generating function $F(t)=\sum_{n>0} f_{n} t^{n}$ is algebraic, if there is a nontrivial polynomial $P$ in two variables, such that $P(F(t), t)=0$. Otherwise, it is transcendental.

In 2] Mireille Bousquet-Mélou conjectured the following:

Conjecture 1.1. Consider the generating function for walks in the slit plane with a given set of steps $\mathfrak{S}$, counted according to their length and their end-coordinates:

$$
S(x, y ; t)=\sum_{\begin{array}{c}
\text { Walk on the slit plane } \\
\text { starting at the origin } \\
\text { with steps in } \mathfrak{S}
\end{array}} t^{\text {length } W} x^{\mathrm{x}-\mathrm{final} W} y^{\mathrm{y}-\mathrm{final} W} .
$$

Suppose that the set of steps is not degenerated and thus all four quadrants of the plane can be reached by some walk, and that the greatest common divisor of the vertical parts of the steps is equal to one.

Then this generating function is algebraic in $t$, if and only if the height of any step is at most one.

In fact, she proved one part of this conjecture in Section 7 of the above paper, namely, that walks with steps that have height at most one have an algebraic

LaBRI, Université Bordeaux I, Research financed by EC's IHRP Programme, within the Research Training Network "Algebraic Combinatorics in Europe", grant HPRN-CT-2001-00272. martin.rubey@labri.fr

http://www.mat.univie.ac.at/〜rubey 


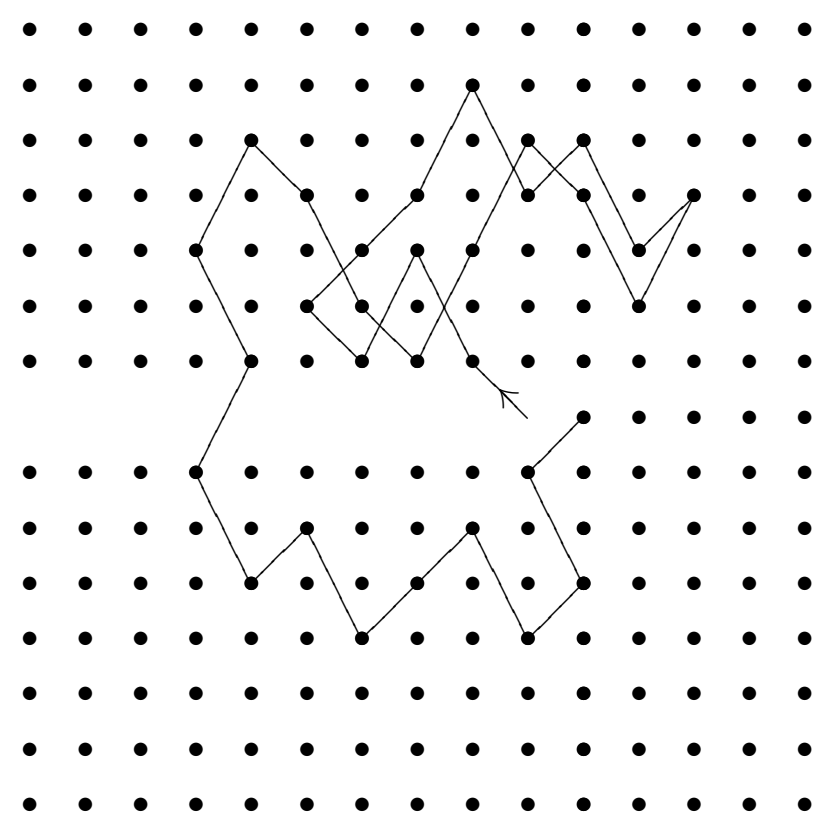

Figure 1. A walk on the slit plane

generating function. Furthermore, in Section 8 she proved for one family of stepsets that the corresponding generating functions have to be transcendental. In the present paper, we prove the following:

Theorem 1.2. Let $\mathcal{H}$ and $\mathcal{V}$ be two finite sets of integers, the greatest common divisor of the integers in each set being equal to one. Furthermore, assume that both of the sets $\mathcal{H}$ and $\mathcal{V}$ contain positive and negative numbers, and that $\mathcal{V}$ contains an element with absolute value at least 2 . Finally, assume that the minimum of $\mathcal{V}$ is at least -2 .

Let $\mathfrak{S}$ be the Cartesian product of the two sets: $\mathfrak{S}=\mathcal{H} \times \mathcal{V}$, where $\mathcal{H}$ is the horizontal and $\mathcal{V}$ is the vertical part of the steps. Then the following generating functions for walks in the slit plane with set of steps $\mathfrak{S}$ are transcendental in $t$ :

- the generating function $S_{i, 0}(t)$ for walks ending at a prescribed coordinate $(i, 0)$,

- the generating function $L(t)$ for loops, i.e., walks that return to the origin,

- the generating function $S_{0}(1 ; t)$ for walks ending anywhere on the $x$-axis, and

- $S(1,1 ; t)$, which is the generating function for walks ending anywhere in the slit plane.

For example, the set of steps of the walk in Figure 1 is the Cartesian product of $\mathcal{H}=\{-1,+1\}$ and $\mathcal{V}=\{-2,+1,+2\}$.

In fact we consider a slightly more general problem: we allow the steps in $\mathcal{H}$ and $\mathcal{V}$ to be weighted with positive real numbers. The weight of a step in the product set $\mathfrak{S}=\mathcal{H} \times \mathcal{V}$ then is the product of the weights of its corresponding vertical and horizontal parts and the weight of a walk is the product of the weights of its individual steps. 
As in 2], we will use a special case of the following theorem to determine in which cases the generating function cannot be algebraic:

Theorem 1.3. 4 Let $F(t)$ be an algebraic function over $\mathbb{Q}$ that is analytic at the origin, then its $n^{\text {th }}$ Taylor coefficient $f_{n}$ has an asymptotic equivalent of the form

$$
f_{n}=\frac{\beta^{n} n^{s}}{\Gamma(s+1)} \sum_{i=0}^{m} C_{i} \omega_{i}^{n}+O\left(\beta^{n} n^{t}\right),
$$

where $s \in \mathbb{Q} \backslash\{-1,-2,-3, \ldots\}, t<s ; \beta$ is a positive algebraic number and the $C_{i}$ and $\omega_{i}$ are algebraic with $\left|\omega_{i}\right|=1$.

It follows easily that an algebraic function cannot have an appearance of a negative integer power of $n$ anywhere in the full asymptotic expansion of its Taylor coefficients.

\section{AN EXPRESSION FOR THE GENERATING FUNCTION FOR WALKS ON THE SLIT}

PLANE

The fundamental theorem for walks on the slit plane is the following:

Theorem 2.1. (Proposition 9 in [2]) Let

$$
B(x ; t)=\sum_{\begin{array}{c}
W \text { walk on } \mathbb{Z}^{2} \\
\text { starting at the origin } \\
\text { ending on the } x \text {-axis } \\
\text { with steps in } \mathfrak{S}
\end{array}} x^{\mathrm{x}-\mathrm{final}(W)} t^{\operatorname{length}(W)}
$$

be the generating function for bilateral walks, that is, walks that end on the $x$-axis but are otherwise unconstrained.

For $i \geq 1$, the generating function $S_{i, 0}(t)$ for walks on the slit plane ending at $(i, 0)$ can be computed by induction on $i$ via the following identity:

$$
\sum_{k=1}^{i} \frac{(-1)^{k-1}}{k} \sum_{\substack{i_{1}+i_{2}+\ldots+i_{k}=i \\ i_{1}>0, i_{2}>0, \ldots, i_{k}>0}} S_{i_{1}, 0}(t) S_{i_{2}, 0}(t) \ldots S_{i_{k}, 0}(t)=\left[x^{i}\right] \log B(x ; t) .
$$

Note that it follows that $\log B(x ; t)$ has positive Taylor coefficients.

Now we can take advantage of the special structure of the set step $\mathfrak{S}$. Since it decomposes into a horizontal and a vertical part, the generating function for bilateral walks factorises:

$$
B(x ; t)=\bar{B}(H(x) t)
$$

where

$$
\bar{B}(t)=\sum_{\begin{array}{c}
W \text { walk on } \mathbb{Z} \\
\text { from } 0 \text { to } 0 \\
\text { with steps in } \mathcal{V}
\end{array}} t^{\text {length }(W)}
$$

is the generating function for bridges and

$$
H(x)=\sum_{h \in \mathcal{H}} x^{h}
$$

is the step (Laurent-)polynomial for $\mathcal{H}$. 


\section{Transcendence of $\left[x^{i}\right] \log B(x ; t)$}

In this section we show that $\left[x^{i}\right] \log B(x ; t)$ cannot be algebraic if the set $\mathcal{V}$ contains an element with absolute value strictly greater than one.

To this end, we consider the asymptotic expansion of $\left[t^{n} x^{i}\right] \log B(x ; t)$. Since $B(x ; t)$ factorises, we have

$$
\begin{aligned}
{\left[t^{n} x^{i}\right] \log B(x ; t) } & =\left[t^{n} x^{i}\right] \log \bar{B}(H(x) t) \\
& =\left[x^{i}\right](H(x))^{n}\left[t^{n}\right] \log \bar{B}(t) .
\end{aligned}
$$

Therefore, we have divided the problem in two: we will show that the asymptotic expansions of both $\left[x^{i}\right](H(x))^{n}$ and $\left[t^{n}\right] \log \bar{B}(t)$ contain a term $n^{-k / 2}$ for some odd $k$.

3.1. Asymptotics of the horizontal part. Let min be the minimal integer such that $H(x) x^{\min }$ is a polynomial. To determine the asymptotics of $\left[x^{i}\right](H(x))^{n}=$ $\left[x^{n \cdot m i n}\right] x^{-i}\left(H(x) x^{m i n}\right)^{n}$, we can use the following theorem:

Theorem 3.1 (3, 5]). Let $g(z)$ be an analytic function of degree $d$ with positive coefficients assumed to be aperiodic and such that $g(0) \neq 0$, and let $a(z)$ be analytic except possibly at zero, where a pole is allowed. Let $\lambda$ be a positive number of some subinterval $\left[\lambda_{a}, \lambda_{b}\right]$ of the open interval $] 0, d[$. Then, with $N=\lfloor\lambda n\rfloor$, one has uniformly for $\lambda \in\left[\lambda_{a}, \lambda_{b}\right]$

$$
\left[z^{N}\right] a(z)(g(z))^{n}=a(\zeta) \frac{(g(\zeta))^{n}}{\zeta^{N+1} \sqrt{2 \pi n R}}(1+o(1)),
$$

where $\zeta$ is the unique positive root of the equation

$$
\zeta \frac{g^{\prime}(\zeta)}{g(\zeta)}=\lambda
$$

and

$$
R=\frac{d^{2}}{d \zeta^{2}}[\log g(\zeta)-\lambda \log \zeta]
$$

Let $p=\operatorname{gcd}\{k+\min : k \in \mathcal{H}\}$ and note that in the case $p>1$, every $p^{\text {th }}$ coefficient of $(H(x))^{n}$ will be zero. Thus, we take $g(x)=H\left(x^{1 / p}\right) x^{\min / p}$, and $a(x)=x^{-i / p}$, and use the theorem to determine $\left[x^{n \cdot \min / p}\right] a(x)(g(x))^{n}$. In this situation, $\lambda=\mathrm{min} / p$ is constant, therefore $\zeta$ and $R$ must be constant, too. Hence, the asymptotic expansion of $\left[x^{i}\right](H(x))^{n}$ contains an appearance of $n^{-1 / 2}$.

3.2. Asymptotics of the vertical part. To determine the asymptotic behaviour of $\left[t^{n}\right] \log \bar{B}(t)$ we will use analysis of singularities. In a first step, we have to determine the singularities of the expression. Following the general theory of singularity analysis, all the contributions from these singularities must be added up.

According to the remark after Theorem 2.1 and the factorisation (11), we have that $\left[t^{n}\right] \log \bar{B}(t)$ is always positive. Therefore we can apply Pringsheim's theorem:

Theorem 3.2. 6] If a function with a finite radius of convergence has Taylor coefficients that are nonnegative, then one of its singularities of smallest modulus - a dominant singularity - is real positive. 
Since the logarithm is singular only at the origin, and $\bar{B}(t)$ is strictly positive for positive real numbers, Pringsheim's theorem implies that one dominant singularity of $\log \bar{B}(t)$ is in fact a singularity - call it $\rho-$ of $\bar{B}(t)$. Of course, there can be other dominant singularities of $\log \bar{B}(t)$ arising from singularities of $\bar{B}(t)$. We will discuss them below.

Furthermore, it might happen - although we believe that it does not - that $\bar{B}(t)$ has zeros on the circle around the origin with radius $\rho$, thus also making $\log \bar{B}(t)$ singular. However, such singularities can never contribute a summand of order $n^{-k / 2}$ for odd $k$, so we can simply ignore them. Note that $\bar{B}(t)$ cannot vanish for $|t|<\rho$, since $\rho$ is a dominant singularity of $\log \bar{B}(t)$.

Now we want to compute the contribution of the dominant singularities of $\bar{B}(t)$ to the asymptotic expansion of $\log \bar{B}(t)$. To do so, we need a better understanding of $\bar{B}(t)$, which is the generating function for bridges with step set $\mathcal{V}$.

Luckily, this generating function has already been studied. We define the step (Laurent-)polynomial for $\mathcal{V}$ as

$$
V(y)=\sum_{v \in \mathcal{V}} y^{v}
$$

and the characteristic curve determined by $\mathcal{V}$ by the equation

$$
1-t V(y)=0 \quad \text { or equivalently } \quad y^{\min _{v}}=t\left(y^{\min _{v}} V(y)\right)=0,
$$

where $\min _{v}=-\min \mathcal{V}$ is the minimal integer to make the equation polynomial.

We say that the functional equation (2) is reduced, if the greatest common divisor of the exponents of the monomials in $V(y)$ is equal to one, which is one of the assumptions in our main Theorem 1.2

We say that the functional equation (2) has period $p$, if the greatest common divisor of the exponents of the monomials in $y^{\min _{v}} V(y)$ is equal to $p$.

As is well known, the period is also the number of dominant singularities of $\bar{B}(t)$, which are all conjugate to the real dominant singularity $\rho$. In our case however, it can be seen (11, Section 3.3]) that the asymptotic formula for bridges is obtained from the asymptotic expansion derived from the singularity at $\rho$ by multiplying with $p$. Since we are only interested in the presence or absence of a term $n^{-k / 2}$ for some odd $k$ in the asymptotic expansion, we can assume from now on that the functional equation (2) is aperiodic, i.e., has period one.

It can be seen 1, 6 that the solutions of this functional equation organise themselves into "small" and "large" branches. Here, "small" means that the solution $y(t)$ tends to zero as $t$ tends to zero, whereas "large" means that $y(t)$ tends to infinity as $t$ approaches zero.

It is only the set of "small" solutions that is interesting for us, and it can be seen - using a limit case of Pellet's Theorem, see for example [7] - that there are $\min _{v}$ of them. A nice expression for the generating function for bridges is given by the following theorem:

Theorem 3.3. 1, Theorem 1 and proof of Theorem 3] The generating function for bridges is an algebraic function given by 


$$
\begin{aligned}
\bar{B}(t) & =t \sum_{j=1}^{\min _{v}} \frac{y_{j}^{\prime}(t)}{y_{j}(t)}=t \frac{d}{d t} \log \left(y_{1}(t) y_{2}(t) \cdots y_{\text {min }_{v}}(t)\right) \\
& =\frac{1}{2 \pi i} \int_{|y|=\tau} \frac{d y}{y(1-t V(y))},
\end{aligned}
$$

where the expressions involve all the small branches $y_{1}, y_{2}, \ldots, y_{\min _{v}}$ of the characteristic curve (2), and $\rho$ is the radius of convergence of $\bar{B}(t)$. Furthermore, the principal branch $y_{1}(t)$, i.e., the branch with real coefficients, has a square root singularity at $\rho$ and the product of all the other small branches is analytic for all $t$ with $|t| \leq \rho$. More precisely, $\rho$ is given by $\rho=1 / V(\tau)$, where $\tau$ is the unique positive number with $V^{\prime}(\tau)=0$.

Thus, applying the Newton-Puiseux theorem, we can develop $\bar{B}(t)$ around the singularity $\rho$, setting $\tilde{t}=\sqrt{\rho-t}$ :

$$
\bar{B}(t)=a_{-1} / \tilde{t}+a_{0}+a_{1} \tilde{t}+a_{2} \tilde{t}^{2}+\cdots
$$

Composing this expansion with the Taylor expansion of the logarithm we obtain

$$
\log \bar{B}(t)=\log a_{-1} / \tilde{t}+\log \left(1+\frac{a_{0}}{a_{-1}} \tilde{t}+\frac{a_{1}}{a_{-1}} \tilde{t}^{2}+\cdots\right) .
$$

Now we want to find a term $n^{-k / 2}$ for some odd $k$ in the asymptotic expansion of the coefficients of the above series. Clearly,

$$
\left[t^{n}\right] \log a_{-1} / \tilde{t}=\left[t^{n}\right] \log a_{-1} / \sqrt{\rho-t} \sim c_{0} \rho^{-n} n^{-1},
$$

for some constant $c_{0}$, is not what we are looking after. However, we have

$$
\log \left(1+\frac{a_{0}}{a_{-1}} \tilde{t}+\frac{a_{1}}{a_{-1}} \tilde{t}^{2}+\cdots\right)=\frac{a_{0}}{a_{-1}} \tilde{t}+\cdots
$$

and

$$
\left[t^{n}\right] \frac{a_{0}}{a_{-1}} \tilde{t}=\left[t^{n}\right] \frac{a_{0}}{a_{-1}} \sqrt{\rho-t} \sim c_{1} \rho^{-n} n^{-3 / 2},
$$

for some constant $c_{1}$. Provided that $a_{0}$ does not vanish, this term will guarantee transcendence of the generating function for walks on the slit plane. Thus we need the constant term in the singular expansion of $\bar{B}(t)$.

Since the product of the non-principal branches $y_{2}(t) y_{3}(t) \ldots y_{\min }(t)$ is analytic and non-zero at $\rho$, the contribution of $t \frac{d}{d t} \log \left(y_{2}(t) \cdots y_{\text {min }_{v}}(t)\right)$ to the constant term in the singular expansion of $\bar{B}(t)$ around $\rho$ is the sum of the residues of $1 / y(1-\rho V(y))$ at the zeros of $1-\rho V(y)$ that are strictly smaller than $\tau$ in modulus.

To obtain the contribution of $t \frac{d}{d t} \log \left(y_{1}(t)\right)$, we proceed as follows:

$$
\begin{aligned}
& {\left[\tilde{t}^{0}\right] t \frac{d}{d t} \log \left(y_{1}(t)\right)} \\
& =\left[\tilde{t}^{0}\right]\left(\rho-\tilde{t}^{2}\right)\left(-\frac{1}{2 \tilde{t}}\right) \frac{d}{d \tilde{t}} \log \left(y_{1}(t)\right) \\
& =-\frac{\rho}{2}[\tilde{t}] \frac{d}{d \tilde{t}} \log \left(y_{1}(t)\right) \\
& =\rho\left[\tilde{t}^{2}\right] \log \left(y_{1}(t)\right) .
\end{aligned}
$$


To obtain the coefficient of $\tilde{t}^{2}$ in $z=\log \left(y_{1}(t)\right)$, we consider the Taylor expansion of $0 \equiv G(t, z)=1-t V\left(e^{z}\right)$ around $(\rho, \log \tau)$, where $\tau=y_{1}(\rho)$. We set $\tilde{z}=z-\log \tau$ and write $G$ short for $G(\rho, \log \tau)$, subscripts denote the partial derivative:

$$
\begin{aligned}
0 & =G(t, z) \\
& =G-G_{t} \tilde{t}^{2}+G_{z} \tilde{z}-G_{t, z} \tilde{t}^{2} \tilde{z}+\frac{1}{2} G_{z, z} \tilde{z}^{2}-\frac{1}{2} G_{t, z, z} \tilde{t}^{2} \tilde{z}^{2}+\frac{1}{6} G_{z, z, z} \tilde{z}^{3}+\ldots,
\end{aligned}
$$

since $G_{t, t} \equiv 0$. We have

$$
\begin{aligned}
G & =0 \\
G_{t} & =-\left.V\left(e^{z}\right)\right|_{(t=\rho, z=\log \tau)}=-\frac{1}{\rho} \\
G_{z} & =-\left.t e^{z} V^{\prime}\left(e^{z}\right)\right|_{(t=\rho, z=\log \tau)}=-\rho \tau V^{\prime}(\tau)=0 \\
G_{t, t} & =0 \\
G_{t, z} & =-\left.e^{z} V^{\prime}\left(e^{z}\right)\right|_{(t=\rho, z=\log \tau)}=0 \\
G_{z, z} & =-\left.t\left(e^{2 z} V^{\prime \prime}\left(e^{z}\right)+e^{z} V^{\prime}\left(e^{z}\right)\right)\right|_{(t=\rho, z=\log \tau)}=-\rho \tau^{2} V^{\prime \prime}(\tau) \\
G_{z, z, z} & =-\rho \tau^{3} V^{\prime \prime \prime}(\tau)-\rho \tau^{2} V^{\prime \prime}(\tau) .
\end{aligned}
$$

Therefore, substituting into the Taylor expansion $\tilde{z}=\alpha \tilde{t}+\beta \tilde{t}^{2}+O\left(\tilde{t}^{3}\right)$ we obtain

$$
\begin{aligned}
0 & =-G_{t} \tilde{t}^{2}+\frac{1}{2} G_{z, z} \tilde{z}^{2}+\frac{1}{6} G_{z, z, z} \tilde{z}^{3}+O\left(\tilde{t}^{4}\right) \\
& =\left(-G_{t}+\frac{1}{2} G_{z, z} \alpha^{2}\right) \tilde{t}^{2}+\left(G_{z, z} \alpha \beta+\frac{1}{6} G_{z, z, z} \alpha^{3}\right) \tilde{t}^{3}+O\left(\tilde{t}^{4}\right) .
\end{aligned}
$$

Thus

$$
\begin{aligned}
& \alpha=\sqrt{\frac{2 G_{t}}{G_{z, z}}}=\frac{1}{\rho \tau} \sqrt{\frac{2}{V^{\prime \prime}(\tau)}} \\
& \beta=-\frac{\alpha^{2} G_{z, z, z}}{6 G_{z, z}}=-\frac{\tau V^{\prime \prime \prime}(\tau)+3 V^{\prime \prime}(\tau)}{3\left(\rho \tau V^{\prime \prime}(\tau)\right)^{2}} .
\end{aligned}
$$

It is easy to check that $\beta$, i.e., the coefficient of $\tilde{t}^{0}$ in the singular expansion of $t \frac{d}{d t} \log \left(y_{1}(t)\right)$ is exactly one half of the residue of $1 / y(1-\rho V(y))$ at $\tau$.

In summary, we have shown the following:

Theorem 3.4. Consider bridges with set of steps $\mathcal{V}$. Let $y_{1}(t), y_{2}(t), \ldots, y_{\text {min }_{v}}(t)$ be the solutions of the functional equation $1-t \sum_{v \in \mathcal{V}} y^{v}=0$ that tend to zero as $t$ goes to zero, $y_{1}(t)$ being the branch with real positive Taylor coefficients. Let $\tau$ be the unique positive real number with $V^{\prime}(\tau)=0$ and let $\rho=\frac{1}{V(\tau)}$. Furthermore, let $\tau_{k}=y_{k}(\rho)$ for $k \in\left\{2,3, . ., \min _{v}\right\}$.

Then the constant term in the singular expansion of the generating function for bridges is

$$
\frac{1}{2} \operatorname{res}_{y=\tau} \frac{1}{y(1-\rho V(y))}+\sum_{k=2}^{\min _{v}} \operatorname{res}_{y=\tau_{k}} \frac{1}{y(1-\rho V(y))} .
$$

Unfortunately, we were not able to show that this expression does not vanish if the step set contains a step of height strictly greater than one. However, we have the following conjecture, that we are able to prove partially for some special cases: 
Conjecture 3.5. Let $V(y)$ be a Laurent-polynomial with positive coefficients with highest exponent equal to $\max _{v}$ and lowest exponent equal to $-\min _{v}$. Let $\tau$ be the unique positive solution of $V^{\prime}(\tau)=0$ and

$$
f_{<}(y)=\prod_{\substack{V(\kappa)=V(\tau) \\|\kappa|<\tau}}(y-\kappa)=\sum_{k=0}^{\min _{v}-1} a_{k} z^{k}
$$

and

$$
f_{>}(y)=\prod_{\substack{V(\kappa)=V(\tau) \\|\kappa|>\tau}}(y-\kappa)=\sum_{k=0}^{\max _{v}-1} b_{k} z^{k}
$$

Consider the decomposition

$$
\frac{1}{y(V(y)-V(\tau))}=\frac{\alpha+\beta y}{(y-\tau)^{2}}+\frac{p_{<}(y)}{f_{<}(y)}+\frac{p_{>}(y)}{f_{>}(y)}
$$

where the degree of $p_{<}$is $\min _{v}-2$ and the degree of $p_{>}$is $\max _{v}-2$.

Then

$$
\begin{aligned}
& 0<a_{0}<a_{1}<\cdots<a_{\text {min }_{v}-1} \\
& 0<b_{\max _{v}-1}<b_{\max _{v}-2}<\cdots<b_{0},
\end{aligned}
$$

the leading term of $p_{<}$is negative if $\min _{v}>1$ and the leading term of $p_{>}$is positive if $\max _{v}>1$.

The negativity of the leading term of $p_{<}$in the conjecture would already imply that the constant term of the singular expansion of $\bar{B}(t)$ around $\rho$ does not vanish: $-\frac{1}{\rho}\left(\frac{\beta}{2}+\left[y^{\min _{v}-2}\right] p_{<}(y)\right)$ is exactly the value given by (44). Since replacing $y$ by $1 / y$ in the Laurent-polynomial $V(y)$ changes the sign of $\beta$ in the decomposition (5), we can assume that $\beta$ is negative or zero. In fact, if $\min _{v}=1$, it follows from (3) that $\beta$ is negative. Since $\left[y^{\min _{v}-2}\right] p_{<}(y)<0$ for $\min _{v}>1$, the claim follows.

We can prove parts of the conjecture for $\min _{v} \leq 3$ : In general, it is easy to see that the product of $f_{<}$and $f_{>}$has positive coefficients and that both of their constant terms must be positive.

If $\min _{v} \leq 2$ we can show (6) and (7) by inductive arguments. We were also able to check the case $\min _{v}=3$ and $\max _{v} \leq 4$.

If $\min _{v}=2$ we can also show that $\left[y^{\min _{v}-2}\right] p_{<}(y)<0$ : in this case, $p_{<}(y)$ is constant and equals $1 /\left(\tau_{2} V^{\prime}\left(\tau_{2}\right)\right)$, where $\tau_{2}$ is the only negative zero of $V(y)=V(\tau)$ which is smaller than $\tau$ in modulus. Since $V(y)$ tends to infinity as $y$ approaches $0-$, we have that $V^{\prime}\left(\tau_{2}\right)>0$, which implies the claim.

Finally, if $\min _{v}=2$ and the coefficients of $V$ are either zero or one, we can also conclude that $\beta$ is negative: in this case the numerator of (3) equals

$$
-\tau V^{\prime \prime \prime}(\tau)-3 V^{\prime \prime}(\tau)+3 \tau^{-1} V^{\prime}(\tau)=-3 a_{-1} \tau^{-3}+3 a_{1} \tau^{-1}-15 a_{3} \tau+\ldots
$$


If $a_{1}=0$ then the above expression is trivially negative. Otherwise we have to show that $3 \tau^{-1}<15 \tau$. We show that $\tau>\frac{1}{2}$, which is sufficient: We have

$$
\begin{aligned}
V^{\prime}(y) & =-2 y^{-3}+\sum_{k \geq-1} k a_{k} y^{k-1} \\
& \leq-2 y^{-3}+\frac{1}{(1-y)^{2}}
\end{aligned}
$$

which is negative for $y \leq \frac{1}{2}$.

\section{Transcendence}

It is now a simple matter to complete the proof of the main Theorem 1.2 Since in the circumstances of the theorem the asymptotic expansion of

$$
\left[t^{n} x^{i}\right] \log B(x ; t)=\left[x^{i}\right](H(x))^{n}\left[t^{n}\right] \log \bar{B}(t)
$$

contains a term $n^{-2}$, the series $\left[x^{i}\right] \log B(x ; t)$ cannot be algebraic. When $i$ is minimal such that there is at least one walk in the slit plane with steps in $\mathfrak{S}$ ending at $(i, 0)$, Theorem 2.1 gives that $\left[x^{i}\right] \log B(x ; t)$ is the generating for such walks. To settle the transcendence of $S_{i, 0}(t)$ for general $i$, we only need to note that $\left[t^{n}\right] \log B(x ; t) \sim c_{0} \rho^{-n} n^{-1}$, where, as we proved in the last section, $c_{0}=\sqrt{\frac{2 G_{t}}{G_{z, z}}}=$ $\frac{1}{\rho \tau_{k}} \sqrt{\frac{2}{V^{\prime \prime}\left(\tau_{k}\right)}}$, and thus does not vanish. Hence, the leading term of $\left[t^{n} x^{i}\right] \log B(x ; t)$ contains a factor of $1 / n^{3 / 2}$. Thus, in the convolution formula for $S_{i, 0}(t)$, the term $1 / n^{2}$ in the asymptotic expansion of $\left[x^{i}\right] \log B(x ; t)$ cannot be cancelled by terms of the asymptotic expansion of the product of two or more functions $S_{i_{j}, 0}$.

The proof of the non-D-finiteness of the other functions can be copied verbatim from the proof of Proposition 22, page 282 of [2].

\section{Acknowledgements}

I would like to thank Michael Drmota, Bernhard Gittenberger, Bernhard Lamel and Bodo Laß for numerous stimulating discussions concerning the nature of the solutions of the functional equation (2). Also I'm very grateful for two anonymous referees who pointed out numerous mistakes and a wrong conjecture appearing in the manuscript. And, of course, I would like to thank Mireille Bousquet-Mélou for introducing me to the problem and for a wonderful stay in Bordeaux.

\section{REFERENCES}

1. Cyril Banderier and Philippe Flajolet, Basic analytic combinatorics of directed lattice paths, Theoret. Comput. Sci. 281 (2002), no. 1-2, 37-80, Selected papers in honour of Maurice Nivat. MR 2003g:05006

2. Mireille Bousquet-Mélou, Walks on the slit plane: other approaches, Advances in Applied Mathematics 27 (2001), no. 2-3, 243-288, Special issue in honor of Dominique Foata's 65th birthday (Philadelphia, PA, 2000). MR 2002j:60076

3. Michael Drmota, A bivariate asymptotic expansion of coefficients of powers of generating functions, European Journal of Combinatorics 15 (1994), no. 2, 139-152. MR 94k:05014

4. Philippe Flajolet, Analytic models and ambiguity of context-free languages, Theoretical Computer Science 49 (1987), no. 2-3, 283-309, Twelfth international colloquium on automata, languages and programming (Nafplion, 1985). MR 89e:68067

5. Philippe Flajolet and Robert Sedgewick, The average case analysis of algorithms, 1994.

6. Einar Hille, Analytic function theory. Vol. I, II. 2nd ed. corrected., Chelsea Publishing Company, 1973. 
7. Morris Marden, Geometry of polynomials, Second edition. Mathematical Surveys, No. 3, American Mathematical Society, Providence, R.I., 1966. MR 37 \#1562 